\title{
Neutron measurements from beam-target reactions at the ELISE neutral beam test facility ${ }^{\text {a) }}$
}

\author{
X. Xufei, ${ }^{1, b)}$ M. Nocente, ${ }^{2,3}$ F. Bonomo, ${ }^{4,5}$ P. Franzen, ${ }^{6}$ M. Fröschle ${ }^{6}$ G. Grosso, ${ }^{3}$ F. \\ Grünauer, ${ }^{7}$ R. Pasqualotto, ${ }^{4}$ M. Tardocchi, ${ }^{3}$ T. Fan, ${ }^{1}$ and G. Gorini ${ }^{2,3}$ \\ ${ }^{1}$ State Key Laboratory of Nuclear Physics and Technology, Peking University, Beijing 100871, China \\ ${ }^{2}$ Dipartimento di Fisica "G. Occhialini", Università di Milano-Bicocca, Milano 20216, Italy \\ ${ }^{3}$ Istituto di Fisica del Plasma "P. Caldirola", Milano 20216, Italy \\ ${ }^{4}$ Consorzio RFX, Padova 35100, Italy \\ ${ }^{5}$ Istituto Gas Ionizzati, CNR, Padova 35100, Italy \\ ${ }^{6}$ Max-Planck-Institut fürPlasmaphysik, Garching 84518, Germany \\ ${ }^{7}$ Physics Consulting, Zorneding 85604, Germany
}

\begin{abstract}
Measurements of $2.5 \mathrm{MeV}$ neutron emission from beam-target reactions performed at the ELISE neutral beam test facility are presented in this paper. The measurements are used to study the penetration of a deuterium beam in a copper dump, based on the observation of the time evolution of the neutron counting rate from beam-target reactions with a liquid scintillation detector. A calculation based on a local mixing model of deuterium deposition in the target up to a concentration of $20 \%$ at saturation is used to evaluate the expected neutron yield for comparison with data. The results are of relevance to understand neutron emission associated to beam penetration in a solid target, with applications to diagnostic systems for the SPIDER and MITICA Neutral Beam Injection prototypes
\end{abstract}

\section{INTRODUCTION}

Neutron measurements are gaining increasing importance for fusion studies, where they provide essential information on the fuel ion energy distribution of tokamak plasmas. ${ }^{1-5}$ Based on this experience, $2.5 \mathrm{MeV}$ neutron measurements have also been proposed as diagnostics of the beam homogeneity for the SPIDER and MITICA Neutral Beam Injection (NBI) prototypes in Padua. In these devices, neutron emission will be used to measure the footprint of the deuterium beam when it hits the dump. ${ }^{6}$ Unlike tokamak plasmas, neutrons are here due to deuterium-deuterium (dd) reactions between the beam and the adsorbed deuterons in the target and their level thus depend on deuteron absorption in the beam dump.

In order to investigate such process, we have performed neutron measurements at the ELISE facility of the Max Planck Institut für Plasmaphysik in Garching (Germany). ELISE is a "half size" ITER NBI test facility capable to accelerate negative hydrogen and deuterium ions up to $60 \mathrm{kV}$ and to perform plasma operations of the radio frequency ion source for up to 1 hour. ${ }^{7}$

A calibrated liquid scintillator was used to measure the time evolution of neutron emission from the dump in the first (low radio frequency power) deuterium campaign at ELISE. This was compared to calculations, based on a simulation of the deuterium beam penetration in the dump assuming a local mixing model. ${ }^{8}$

\footnotetext{
${ }^{a}$ Contributed paper published as part of the Proceedings of the 20th Topical Conference on High-Temperature Plasma Diagnostics, Atlanta, Georgia, June, 2014.

b) Author to whom correspondence should be addressed: xiexufei@pku.edu.cn
}

The results were used to validate the model for deuterium implantation in the dump up to a saturation level and to understand the associated neutron emission.

The paper is organized as follows. A description of the experimental equipment precedes the measurement results, which are compared to predictions. The extent of the experimental uncertainties on the calculations is then discussed, with special attention on uncertainties affecting the beam power deposition in the dump. Prospects for future investigations are finally addressed.

\section{EXPERIMENTAL SETUP AND DETECTOR CALIBRATION}

An EJ301A liquid scintillator produced by Scionix, with a $16 \%$ detection efficiency to $2.5 \mathrm{MeV}$ neutrons, was installed in vicinity of one of the two inner walls of the ELISE facility, at a distance of approximately $1.8 \mathrm{~m}$ behind the beam dump, as shown in Fig. 1. The scintillator was coupled to an active base H10580 Hamamatsu photomultiplier tube (PMT), designed to minimize gain shifts, ${ }^{9}$ and was used as global monitor of 2.5 $\mathrm{MeV}$ neutron emission from beam-target reactions between the deuterium beam and the dump. The signal coming from the detector was digitized by means of a $14 \mathrm{bit}, 400 \mathrm{Ms} / \mathrm{s}$ custom digitizer based on the ATCA platform. ${ }^{10}$ The spectrum of the detected radiation was then reconstructed in selected time intervals from charge integration of the digitized waveforms. 


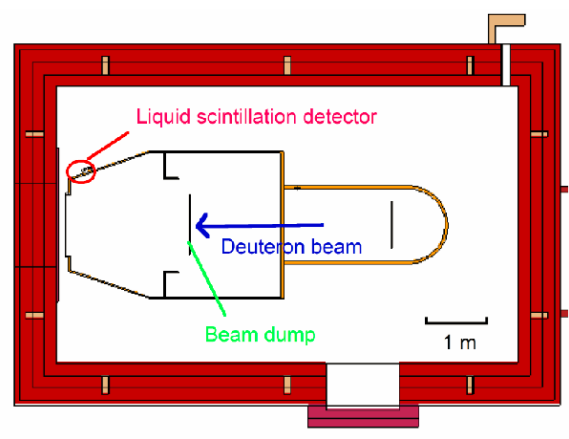

FIG. 1. MCNP model of the ELISE beam facility showing the deuteron beam, the beam dump and position of the liquid scintillation detector used for the measurements reported in this paper.

An important feature of the detector is its capability to distinguish between neutrons and gamma-rays. The latter are an unavoidable background component when performing neutron measurements, as they arise from neutron interactions with materials of the experimental environment. Neutron/gamma-ray discrimination was obtained in the experiment at ELISE by standard methods based on the comparison of charge integration of the digitized waveforms over a long and short gate. ${ }^{11}$ In order to determine the detector response function to $2.5 \mathrm{MeV}$ neutrons, a dedicated calibration experiment was performed at the $4.5 \mathrm{MV}$ tandem Van de Graaff accelerator of Peking University prior to the campaign at ELISE, using quasi monoenergetic neutrons of selected energies between 1.1 and $3 \mathrm{MeV}$, with a $300 \mathrm{keV}$ energy step. Fig. 2 illustrates an example of measured charge integration spectrum for $\mathrm{E}_{\mathrm{n}}=1.4 \mathrm{MeV}$ in the calibration experiment, together with a GEANT4 simulation of the expected detector response, showing good agreement with the measurements. A low energy threshold of $100 \mathrm{keV}$ equivalent electron energy $\left(\mathrm{E}_{\mathrm{ee}}\right)$ was used in the calibration. In the experiment at Peking University, a time of flight technique was adopted to separate neutrons and gammarays and was verified against the detector discrimination capability based on the long/short gate method, without appreciable differences. From the results of the calibration the light output function of the detector was finally determined, which was used in the campaign at ELISE to convert from the measured equivalent electron energy $\left(E_{e e}\right)$ to neutron energy $\left(E_{n}\right)$, for example when setting low energy thresholds in the measured data.

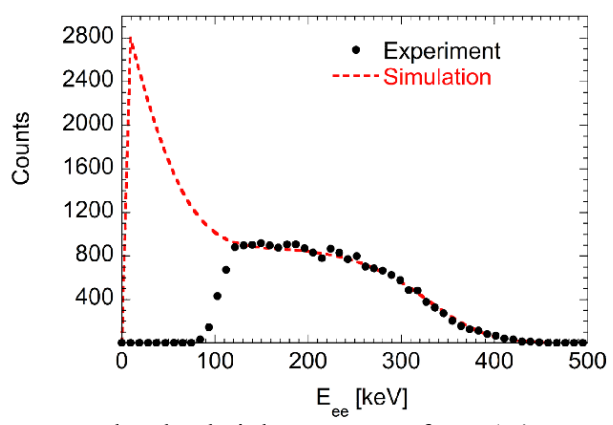

FIG. 2. Measured pulse height spectrum from $1.4 \mathrm{MeV}$ neutrons in the calibration experiment performed at Peking University. $\mathrm{E}_{\mathrm{ee}}$ on the $\mathrm{x}$ axis is the equivalent electron energy. The dashed curve is a simulation with the GEANT4 code.

\section{MEASUREMENT RESULTS AND INTERPRETATION}

Neutron data were taken for the whole first (low radiofrequency power) deuterium campaign at ELISE between January and February 2014. In each pulse, the deuteron beam was accelerated for, generally, $10 \mathrm{~s}$ at a voltage between 25 and 35 $\mathrm{kV}$. The total ion current extracted varied between 4 and $11 \mathrm{~A}$, depending on machine conditioning. An example of a set of measurements provided by our detector for each pulse is illustrated in Fig. 3. The contour plot of the discrimination between neutrons and gamma-rays is shown in Fig. 3(a), where each recorded waveform is represented as a point in the $\mathrm{Q}_{\mathrm{S}} / \mathrm{Q}_{\mathrm{L}}$ plane. By $\mathrm{Q}_{\mathrm{S}}$ and $\mathrm{Q}_{\mathrm{L}}$ we mean here the result of charge integrating each individual event over a short (15 ns) and long (40 ns) gates, respectively. ${ }^{11}$ There is a clear separation between neutrons and gamma-rays, the latter appearing above the discrimination line. From the separated waveforms, we can individually reconstruct the neutron and gamma-ray counting rate evolution during a pulse (Fig. 3(b)). In order to reduce the extent of low energy neutrons in the measured signal, the latter resulting from scattering in the ELISE hall, a threshold of $0.26 \mathrm{MeV}$ (equivalent electron energy) was used in the measurements, corresponding to a neutron energy of $1.15 \mathrm{MeV}$. In this way, the probability of a wrong neutron/gamma-ray event discrimination was reduced to about $5.7 \%$. Here we note that the neutron/gamma-ray time evolution is similar, as expected, and that radiation emission occurs simultaneously with the beam start. The measured neutron/gamma-ray ratio is $\approx 3$.
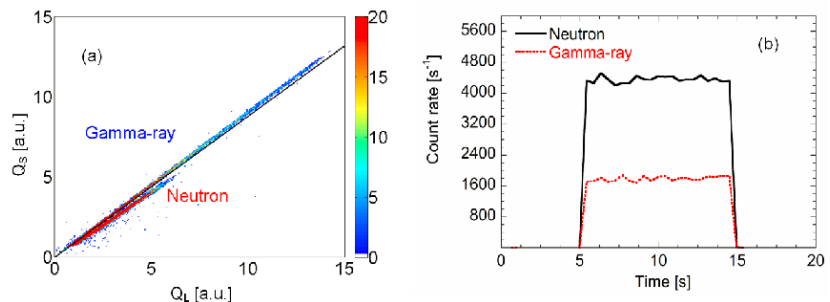

FIG. 3. (a) Neutron/gamma-ray discrimination figure for a typical pulse of the ELISE deuterium campaign. $\mathrm{Q}_{\mathrm{L}}$ and $\mathrm{Q}_{\mathrm{S}}$ are the result of charge integration over a long/short gates used for discrimination. Neutrons and gamma-rays appear on different sides of the oblique black line. (b) Time trace of neutron and gamma-ray emission measured for the same pulse.

The time evolution of the neutron rate for the whole deuterium campaign was built from the measured neutron rate in each pulse as a function of the cumulated deuteron beam time, i.e. the effective time of beam operations, net of pauses (Fig. 4(a), solid curve). Two features can be clearly observed from the data. First, there is an initial phase, lasting about $500 \mathrm{~s}$ (cumulated beam time), when the neutron counting rate increases, up to a value of $\approx 3 \mathrm{kHz}$; then, the counting rate reaches a plateau level. The fluctuations superimposed to this trend come mostly from pulse by pulse variations in the beam current and, to a lesser extent, voltage. Qualitatively, we can explain the initial and plateau phases as follows. The rising part of the curve in Fig. 4(a) corresponds to the buildup of deuterium concentration in the dump, which is manifested as an increasing neutron emission from beam-target reactions. Such process continues until deuterium concentration in the dump reaches saturation. This corresponds to the transition to the plateau phase in the neutron measurements. 

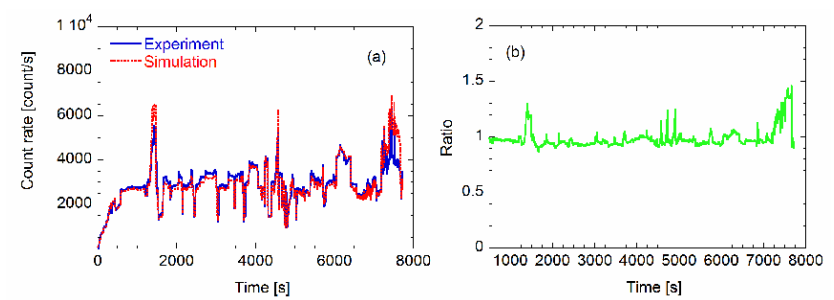

FIG. 4. (a) Measured time evolution of neutron emission for the whole deuterium campaign at ELISE (blue, solid curve). The red dashed curve is the result of a simulation of the expected neutron yield (normalized to data) as a function of time, based on a model of the beam penetration in the target and corresponding neutron emission. (b) Ratio between the experiment and simulation curves of Fig. 4(a) as a function of time at saturation.

In order to interpret quantitatively the data, we have performed a calculation of the neutron yield expected from the target as a function of time, following the model described in $[6$, $12,13]$.

Deuteron deposition in the dump (made by more than $99 \%$ of copper) was simulated using the TRansport of Ion in Matter (TRIM) program. ${ }^{14}$ From the TRIM result, we evaluated the deuteron deposition rate as a function of the penetration depth. This was in turn used to calculate the time evolution of the deuterium concentration $\mathrm{C}(\mathrm{t})$ in copper. The concentration is defined here as the percentage of deuteron to total atoms (both copper and deuteron) per unit volume. To this regard, we assumed a Local Mixing $\operatorname{Model}^{8}$ whereby deuteron migration in copper is neglected and the deuterium concentration cannot exceed a maximum of $20 \% .{ }^{15}$

From the evaluated deuterium density in the dump as a function of depth and time $\mathrm{n}_{\mathrm{D}}(\mathrm{x}, \mathrm{t})$, the local neutron emissivity $y(x, t)=n_{D}(x, t) \cdot \phi \cdot \sigma$ was computed given the known dd energy dependent cross section $\sigma$ and deuterium flux on the dump $\phi$. Besides being a function of depth $\mathrm{x}, \mathrm{y}(\mathrm{x}, \mathrm{t})$ changes at different beam dump positions, as it depends on the beam footprint on the dump. Here we relied on a calculation of the expected power density profile on the dump and considered it to be the same as the deuteron flux profile, which was assumed to be constant as a function of time. The absolute magnitude of the impinging deuteron flux was derived from the measured extracted ionic current, divided by the charge and impinging area.

The calculated time evolution of the deuterium concentration in the ELISE beam dump is shown in Fig. 5 for a position where the beam power deposition is maximum. The corresponding neutron yield as a function of time is also presented. The beam penetrates in the dump up to $\approx 0.5 \mu \mathrm{m}$, which is reflected in the neutron yield as a function of depth. Here we observe that neutrons are emitted only from the first 0.2 $\mu \mathrm{m}$ of copper, due to negligible dd cross section as the deuteron beam loses energy by penetration into the target. Deuterium deposition initially peaks in the middle of the $0.5 \mu \mathrm{m}$ penetration range, becoming more uniform on a time scale of few hundred seconds. Correspondingly, the neutron emission profile has a maximum in the first $0.1 \mu \mathrm{m}$, showing that neutron emission is effectively localized on the surface of the beam dump.
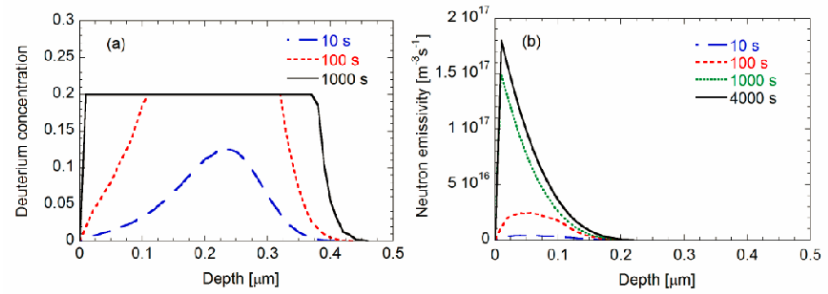

FIG. 5. Calculated profile evolution of deuterium concentration (a) and neutron emissivity (b) in the beam dump of ELISE.

By space integrating the neutron emissivity $y(x, t)$ over the dump surface and depth, we can calculate the total neutron yield $\mathrm{Y}(\mathrm{t})$ as a function of time, which we can compare to measurement (Fig. 4(a), dashed curve). To perform the comparison, the simulation was normalized to data to convert from $\mathrm{Y}(\mathrm{t})$ to the measured counting rate, which also depends on neutron transport from the dump to the detector, besides the scintillator detection efficiency to neutrons. The normalization coefficient was obtained by matching $\mathrm{Y}(\mathrm{t})$ to the initial rising part of the measured counting rate $(t<500 \mathrm{~s})$. From Fig. 4(a), we find that, on a relative scale, the simulation generally reproduces both the observed level of the plateau and the superimposed fluctuations, due to varying ionic current and accelerating voltage, which shows that the local mixing model of beam implantation in the dump adopted for the calculations is able to reproduce the dominant features of the data. A closer inspection reveals however that the agreement between simulation and measurements tends to be weaker at the highest counting rates. This is shown in Fig. 4(b) by the time evolution of the ratio between the simulation and experiment curves of Fig. 4(a) at saturation, and may suggest that physics processes beyond the local mixing model adopted in the calculations (e.g. migration effects and particle escape) could be of relevance at higher beam currents and voltages. For a calculation of the absolute counting rate at the detector position, a MCNP model of ELISE was developed to evaluate neutron transport from the dump to the scintillator. A preliminary run shows that the simulation exceeds measurement by a factor of 2 . Such discrepancy may be due to uncertainties in the neutron transport model and is currently under investigation.

\section{DISCUSSION}

The neutron emission level at ELISE depends on the value of the deuterium concentration $\mathrm{C}_{\mathrm{S}}$ reached at saturation in the dump. In the calculations presented in Fig. 4(a) we have used the value quoted in the literature $(20 \%,[15])$, but it may be of interest to study the sensitivity of our data to variations of $\mathrm{C}_{\mathrm{S}}$. This is illustrated in Fig. 6, where we have repeated the neutron yield calculations for $\mathrm{C}_{\mathrm{S}}=10$ and $30 \%$, besides the $20 \%$ case, which is shown again for reference. As before, each theoretical curve was normalized to experiment by matching calculations to data in the $\mathrm{t}<200 \mathrm{~s}$ region, so that the comparison is on a relative scale. We expect the slope of the rising edge of the count rate curve to be independent from $\mathrm{C}_{\mathrm{S}}$, while the level of plateau would scale linearly. This is confirmed by Fig. 6, which also shows that $\mathrm{C}_{\mathrm{S}}$ should not depart significantly from $20 \%$. This result provides additional confidence on the assumptions used to design neutron diagnostics planned for SPIDER and MITICA ${ }^{6}$. A consideration must however be made on the uncertainties affecting the calculations, particularly those on the deposited 
power P. There are two sources of uncertainty on P. The first one is on the profile of the power deposition, for which we had to rely completely on a calculation, which however showed that the beam deposition involves more than $90 \%$ of the beam dump. A systematic error on the power profile would thus not be too crucial for our model. The reason is that the EJ301 scintillator installed at ELISE, having no line of sight, acts as a global neutron monitor and is thus only marginally sensitive to space variations in the neutron emission from the dump, provided that the overall beam power deposition is unchanged. This was studied in the simulations by comparing the results of Fig. 4(a) with the case of a uniform beam deposition on the dump, at same power. No significant difference in the predicted neutron yield was observed.

Concerning the magnitude of the beam power $\mathrm{P}$ deposited on the dump, a different argument applies. For the yield at saturation $\mathrm{Y}_{\mathrm{S}}$ (plateau) we can expect a proportionality relation $\mathrm{Y}_{\mathrm{S}} \propto \mathrm{P} \cdot \mathrm{C}_{\mathrm{S}}$, that compares to $\mathrm{Y} \propto \mathrm{P}^{2}$ before saturation. The quadratic dependence on $\mathrm{P}$ before saturation derives from the fact that $\mathrm{Y}(\mathrm{t}) \propto \phi \cdot \mathrm{n}_{\mathrm{D}}(\mathrm{t})$, where $\phi \propto \mathrm{P}$ and, similarly, $\mathrm{n}_{\mathrm{D}} \propto \mathrm{P}$. An error on $P$ would be thus manifested quadratically on the rising edge of Fig. 4(a) and, linearly, on the yield at the plateau, affecting our estimation of $\mathrm{C}_{\mathrm{S}}$ in Fig. 6 . In the present experimental setup at ELISE we can estimate an uncertainty of about $20 \%$ on the extracted ionic beam current, which would be similarly reflected on our estimation of $\mathrm{C}_{\mathrm{S}}$. Clearly, a better, model independent determination of the power on the dump would be highly beneficial. Such measurement is not possible at the moment, but may be available in the next deuterium campaigns, thanks to developments in calorimetry systems. ${ }^{16}$ Furthermore, the recent installation of thermocamera diagnostics could provide a model independent map of the beam profile on the dump.

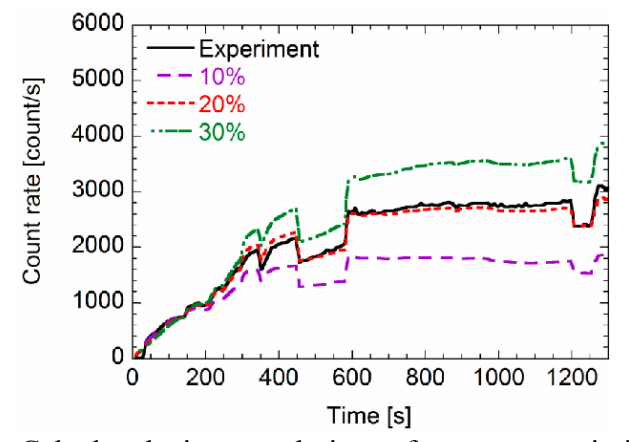

FIG. 6. Calculated time evolution of neutron emission for different values of the deuterium concentration at saturation $C_{S}$ (in percentage; dashed curves) compared to measured data (solid line). The rising slope of each curve is normalized to data of the deuterium build up phase, $\mathrm{t}<200 \mathrm{~s}$. The neutron emission level reached at plateau depends on the assumed concentration level at saturation, with a best match for $\mathrm{C}_{\mathrm{S}}=20 \%$.

Finally, we shall mention the possibility that deuterium, being trapped in copper, is released after a finite time, thus resulting in a lowered neutron yield. This effect was investigated by looking for drops in the measured counting rate of similar set of pulses having a time gap of 3/4 days between each other (typically, before/after a weekend). No evidence was found within error bars. A possible longer term de-trapping of deuterium absorbed in copper will be studied in the future by comparing the neutron yield between consecutive deuterium campaigns separated by a few weeks without operations. Future studies shall also investigate the extent of temperature effects on the deuterium concentration at saturation which, so far, were not observed, but may be manifested at increased power on the calorimeter.

\section{CONCLUSIONS AND OUTLOOK}

Neutron measurements were performed at the ELISE neutral beam test facility with a liquid scintillator used as a global monitor of $2.5 \mathrm{MeV}$ neutron emission from beam-target deuterium-deuterium reactions. The measured counting rate was used to study the evolution of the neutron yield as function of time. A calculation based on the local mixing model and a deuteron concentration of $20 \%$ at saturation was developed and generally found consistent with data on a relative scale and within uncertainties. A discrepancy of a factor two between measurements and a preliminary MCNP simulation was found and is currently under investigation. Future measurements shall be aimed at studying neutron emission in the next high power deuterium campaigns at ELISE, so to determine the relevance of effects beyond the local mixing model at high beam current and also benefitting from model independent measurement of the beam power deposition, thanks to developments in calorimetry systems.

\section{ACKNOWLEDGEMENTS}

This work was carried out with financial support from Fusion for Energy and INFN-Group 5 and has received funding from the Euratom research and training programme 2014-2018 and the National Magnetic Confinement Fusion Science Program of China (Nos. 2013GB106004 and 2012GB101003).

\section{REFERENCES}

${ }^{1}$ Hellesen C et al. 2010 Nucl. Fusion 50084006.

${ }^{2}$ Nocente $\mathrm{M}$ et al. 2010 Nucl. Fusion $\mathbf{5 0} 055001$.

${ }^{3}$ Tardocchi M et al. Plasma Phys. Control. Fusion 55074014.

${ }^{4}$ Nocente $\mathrm{M}$ et al. 2011 Nucl. Fusion 51063011.

${ }^{5}$ Chen Z et al. 2012 Nucl. Fusion 53063023.

${ }^{6}$ Croci $\mathrm{G}$ et al. 2012 Jinst 7 C03010.

${ }^{7}$ Heinemann et al. 2011 Fusion Eng. Des. 86768.

${ }^{8}$ Brice D K, Doyle B L and Wampler W R 1982 J. Nucl. Mat. 111-112 598.

${ }^{9}$ Nocente M et al. 2013 IEEE Trans. Nucl. Sci. 601408.

${ }^{10}$ Nocente M et al. 2010 Rev. Sci. Instrum. 81 10D321.

${ }^{11}$ Yuan Xi et al. 2013 Chinese Physics C 37126001.

${ }^{12}$ Rebai M 2012 "Fast neutron instrumentation for beam diagnostics", chapter 4, PhD Thesis, Università degli Studi di Milano-Bicocca, available online at http://hdl.handle.net/10281/28449.

${ }^{13}$ Doyle B L et al. 1980 J. Nucl. Mat. 93-94 551.

${ }^{14}$ Ziegler J F SRIM/TRIM code, available online at http://www.srim.org.

${ }^{15}$ Kim J 1979 Nucl. Technol. 44315.

${ }^{16}$ Nocentini R et al. 2013 Fusion Eng. Des. 88913. 